\title{
Research Paper \\ Validity and Reliability of the Persian Version of Relationship of Grandchild- Grandparents
}

\author{
Mones Modaresifar ${ }^{1}\left(\mathbb{1}\right.$, Yadollah Abolfathi Momtaz ${ }^{1,2} \oplus$, Ahmad Delbari $^{1}$ (1)
}

1. Iranian Research Center on Aging, University of Social Welfare and Rehabilitation Sciences, Tehran, Iran

2. Malaysian Research Institute on Ageing (MyAgeing), Universiti Putra Malaysia, Serdang, Selangor, Malaysia.

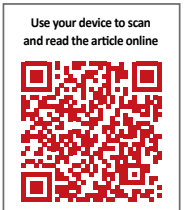

Cftration: Modaresifar M, Abolfathi Momtaz Y, Delbari A. [Validity and Reliability of the Persian Version of Relationship of Grandchild- Grandparents (Persian)]. Iranian Journal of Ageing. 2020; 15(1):42-53. https://doi.org/10.32598/sija.13.10.2030

doi" https://doi.org/10.32598/sija.13.10.2030

Key words:

Reliability, Validity, Grandchildren-grandparents relationship

\section{A B STRACT}

Objectives Studies show that the relationship between Grandchildren and Grandparents affects the health and quality of life of both groups. Currently There is no tool to evaluate the relationship in Iran. The purpose of this study was to assess the validity and reliability of the Persian version of Relationship of Grandchild- Grandparents Elder-Conger.

Methods \& Materials This cross-sectional study was conducted on 505 students aged 9-17 years in Jahrom City in 2018. The Cluster sampling was used to select participants and to collect data. The Internal consistency reliability evaluated by Cronbach's alpha and used Content Validity Index (CVI). Statistical analysis was performed using SPSS V. 23 and AMOS.

Results The study population included the Mean \pm SD of age $12.88 \pm 2.67$ year. The CVI was 0.98 . The questions were approved by experts in terms of being simplified, clear, and understandable. Internal Consistency obtained through Cronbach's alpha was 0.89 ; for emotional dimension of grandchildren toward grandparents was 0.84 ; and for participation dimension was 0.83 . Result of construct validity showed that questionnaire was Fitness. (GFI=0.964, $\mathrm{CMIN}=2.504, \mathrm{AGFI}=0.938$, RMSEA=0.059).

Conclusion The results of the study showed that this questionnaire was of desirable Reliability and Validity. It is suggested that more studies on evaluation reliability and validity are done.

\section{Extended Abstract}

\section{Introduction}

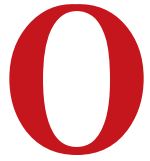

ld age is a natural process of physiological, psychological, and social changes that begins in the embryonic stage and continues until death [1]. The world is rapidly aging as it seeks to increase life expectancy, increase life expectancy, improve health, improve living standards, reduce mortality, and enforce birth control policies. The world's elderly population in 2014 was $12 \%$, which is projected to reach $21 \%$ in 2050 [2]. In Iran, following the government's health policies in the last three decades from 1986-2016, the aging rate has increased from 5.7 to $9.3[3,4]$.

As the elderly population grows, so do their social support, rehabilitation, and health care issues. During this period, age-related physiological and psychosocial changes cause the elderly to be prone to experience negative social consequences at the individual, family, and social stages;

\section{* Corresponding Author:}

Yadollah Abolfathi Momtaz, PhD.

Address: Iranian Research Center on Aging, University of Social Welfare and Rehabilitation Sciences, Tehran, Iran

Tel: +98 (901) 6962134

E-mail: yabolfathi@gmail.com 
therefore, maintaining the quality of life in this period is very important [5]. Studies show that one of the tools for measuring the relationship between grandchildren and grandparents is the Grandparent-Grandchild Relationship Questionnaire used in some foreign studies such as Tan Joe [6] and Khalid Shazed [7].

However, since in Iran only the perspective of grandparents has been studied and on the other hand, the validity of this questionnaire has not been studied so far, this study has evaluated the validity of the Persian version of the Grandparent-Grandchild Relationship Questionnaire.

\section{Methods \& Materials}

This cross-sectional study was performed on 505 male and female students aged 9-17 years studying in the third to eleventh grades of elementary and junior high schools. Students were selected by random cluster sampling. Of inclusion criterion was having at least one grandparent. In the selected schools, with the coordination of the school principal, a full explanation of the purpose of the study was stated. It was mentioned that if the student or his/her parents do not want to participate in the study, they can stop participating, or if after participating in the study, he or his family were unwilling for data analysis, they would be excluded from the study, and thus a passive consent, in which the nature of the study was explained, was obtained from the students' parents or guardian.

Validity and reliability of the questionnaire

\section{Content validity}

In order to determine the Content Validity Index (CVI), 8 geriatricians and psychologists were asked to rate the relevance of each question in the test as follows: Irrelevant 1, Need Serious review 2, Relevant but need review 3, and Completely related 4 .

\section{Face validity}

The main issue in face validity was the subject's understanding of the concept of the test; therefore, the test questions should be selected in terms of words, sentences, and appearance content in a way that stimulates the respondents.

\section{Structural validity}

In this study, to determine the validity of the structure, confirmatory factor analysis was performed using AMOS software V. 23.

\section{Internal reliability}

Cronbach's alpha method, the most common internal stability reliability coefficient, was used in this study, which represents the degree of fit of a group of items that measure a structure and their value is between 0 and 1 . In other words, the alpha value must be at least equal to 0.7 or more [10]. Cronbach's alpha reliability coefficient is useful when the questions are a Likert scale tool that the questions of the Alder-Kunger relation questionnaire were also in the Likert scale.

\section{Study pilot}

The study pilot was conducted on 30 first to eleventh grade students and the questions were assessed for comprehensibility and simplicity. The pilot result showed that the questions were not comprehensible to the first and second grade students and they could not answer the questions due to lack of comprehension, lack of complete independence in answering, and inability to read the questions. Questions and words that were not clear to the students were re-examined and corrected.

\section{Results}

\section{Samples}

The Mean \pm SD age of students participating in the study was $12.88 \pm 2.67$ years. The gender composition of the study participants was $51.1 \%$ female. The highest frequency was in the third and fourth grades of elementary school with $12.5 \%$. About $86.1 \%$ of students had a maternal grandmother, $62.6 \%$ had a maternal grandfather, $68.1 \%$ had a paternal grandmother and $42.2 \%$ had a paternal grandfather. Also, about $5 \%$ of students did not have a grandmother at all and about $25 \%$ of students did not have a grandfather at all.

\section{Content validity}

Content validity Index (CVI) was calculated and the number was 0.98 . The minimum acceptable value for the CVI index was 0.79 and if an item was less than 0.79 should be removed [11]. The values obtained for the CVI showed that the test questions had an acceptable value of the CVI.

\section{Face validity}

All items of the Grandparent-Grandchild Relationship Questionnaire were accepted by students in terms of simplicity, clarity, and comprehensibility. This questionnaire had a good number of questions and was not boring and time consuming for teenagers. According to $12.5 \%$ of ex- 
perts, questions one and three needed serious revision that were revised and corrected.

\section{Structural validity}

Structural validity was performed to show that the questionnaire was two-dimensional, which included emotional and participatory dimensions. AMOS statistical software v. 23 was used. To evaluate the goodness-to-fit index of the model, GFI, AGFI, and RMSEA indices were used and the following results were obtained:

$G F I=0.964, C M I N=2.504, A G F I=0.938, R M S E A=0.059$

Also, the CMIN index was equal to 2.504, which the acceptable value of it is less than 5. The calculated Chisquared had a goodness-to-fit index of the community.

\section{Reliability}

The internal reliability of the questionnaire was calculated using Cronbach's alpha. The Cronbach's alpha value for the emotional dimension of the questionnaire was 0.84 and for the participatory dimension was 0.83 and for the whole questionnaire the relationship was 0.89 , which shows that the Grandparent-Grandchild Relationship Questionnaire had a good internal reliability.

\section{Conclusion}

In order to validate the Grandparent-Grandchild Relationship Questionnaire, content validity, face validity, structural validity, and internal reliability indices were used and the results showed that this questionnaire has good validity and reliability. Content Validity results based on CVI were 0.98 and confirmed [11].

This questionnaire has good Content Validity and, as mentioned before, the minimum acceptable value for the CVI is 0.79 and if the index is less than 0.79 , that item should be removed. The results of face validity showed that the questions were suitable for students in terms of simplicity, clarity, and comprehensibility, and they can answer the questions easily and without problems, and these questions are suitable for ages 9 and up.

The results of construct validity showed that the two emotional and participatory dimensions of the questionnaire had a well goodness-to-fit index and all items were significantly related to their respective factors.
The results of the present study showed that the Persian version of the Grandparent-Grandchild Relationship Questionnaire has good validity and reliability and the answers can be used to examine the views of grandchildren at different ages towards their grandparents. It is suggested that further studies be performed to evaluate the validity of the Grandparent-Grandchild Relationship Questionnaire.

\section{Ethical Considerations}

Compliance with ethical guidelines

This study was approved by the Ethics Committee of University of Social Welfare and Rehabilitation. (Code: IR.USWR.REC.1396.312).

\section{Funding}

This research did not receive any grant from funding agencies in the public, commercial, or non-profit sectors.

\section{Authors' contributions}

Conceptualization: Yadollah Abolfathi Momtaz, Mones Modaresifar; Methodology, analysis, research and review: Mones Modaresifar, Yadollah Abolfathi Momtaz, Ahmad Delbari; writing - review \& editing: Yadollah Abolfathi Momtaz, Mones Modaresifar, Ahmad Delbari; Supervision: Yadollah Abolfathi Momtaz, Ahmad Delbari.

\section{Conflicts of interest}

The authors declared no conflict of interest. 


\title{
اعتبارسنجى نسخه فارسى يرسشنامه رابطه نوه-يدربزرى /مادربزرتى
}

\author{
مونس مدرسى فر' هـ "يدالله ابوالفتحى ممتاز 'به، احمد دلبرى' (1) \\ I. كروه سالمندى، مركز تحقيقات سالمئدى، دانشكاه علوم بهزيستى و توانبخشى، تهران، ايران.

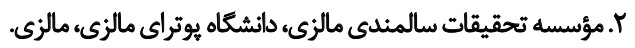

\begin{abstract}
حكיد

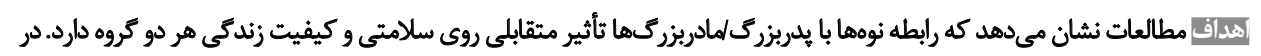

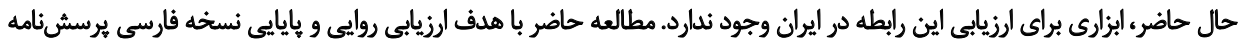

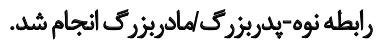

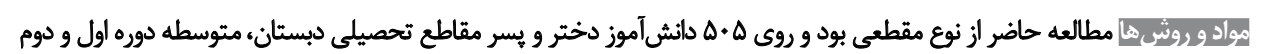

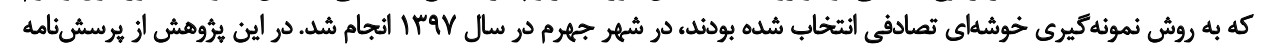

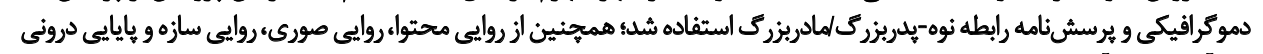

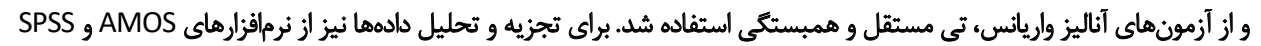

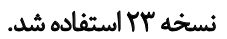

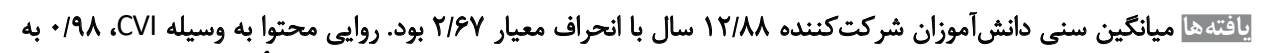

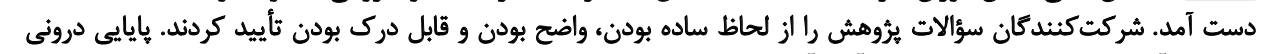

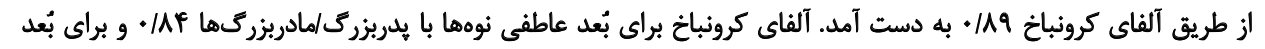

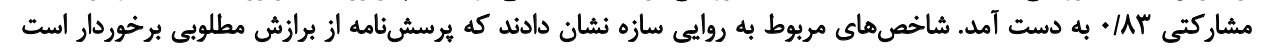

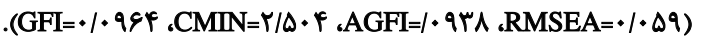

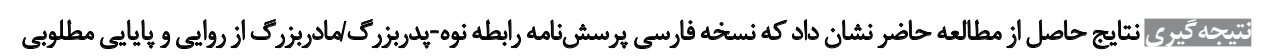

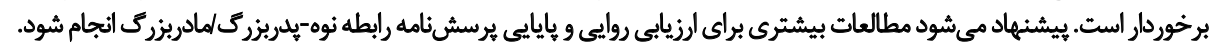

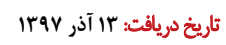

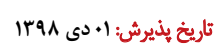

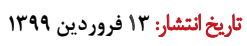

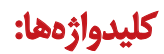 \\ اعتبارسنجى، رابطه

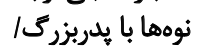 \\ مادبزركها}

سالمندى، تغييرات فيزيولوريك و روانى لجتماعى وابسته به سن

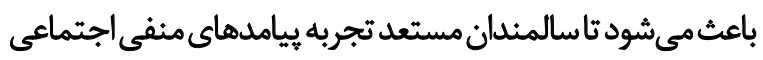

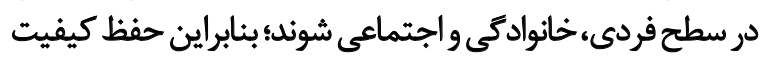

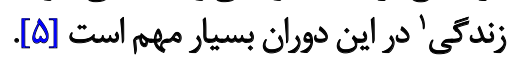

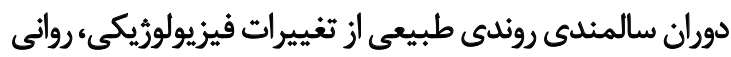

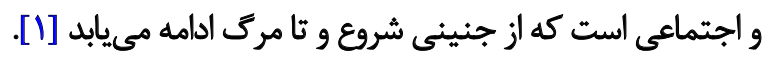

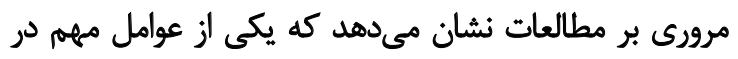

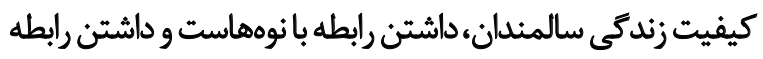

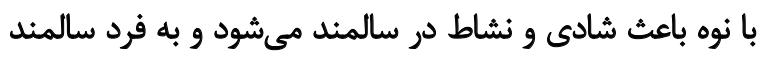

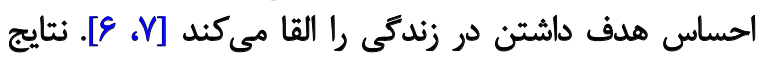

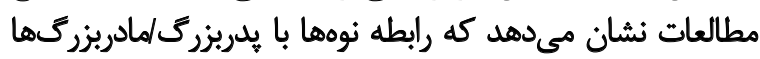

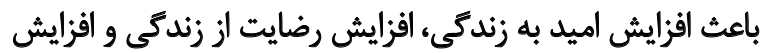

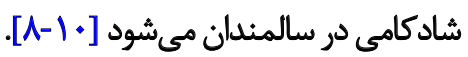

رابطه نوهها با يدربزرك/مادربزركهايشان علاوه بر تأثير در

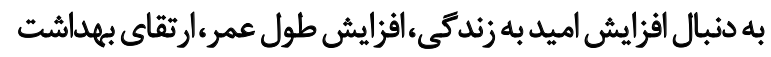

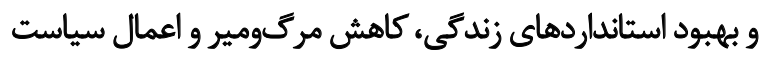

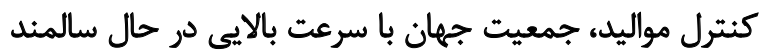

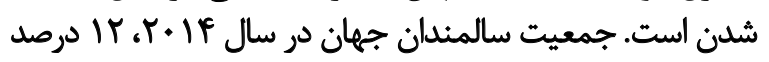

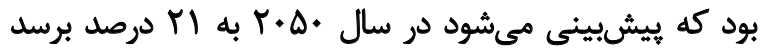

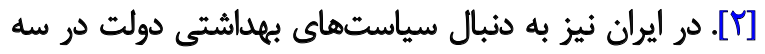

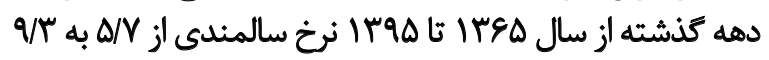
رسيده است [؟، "ז].

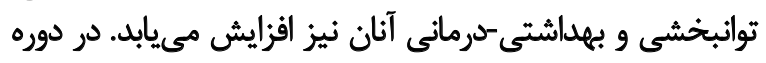

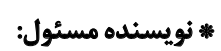

$$
\begin{aligned}
& \text { دكتر يدالله ابوالفتحى ممثتاز }
\end{aligned}
$$

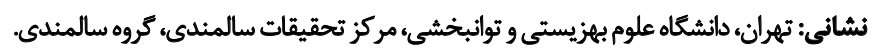

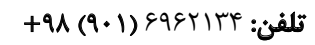

$$
\begin{aligned}
& \text { بست الكترونيكي: yabolfathi@gmail.com }
\end{aligned}
$$




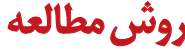

تعيين حجمي نمونه

براى تعيين حجم نمونه از فرمول كوكران

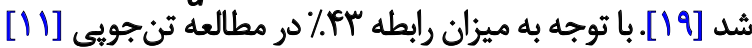

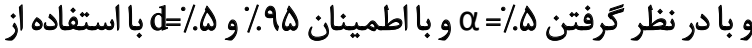

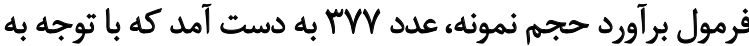

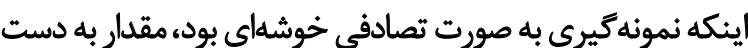

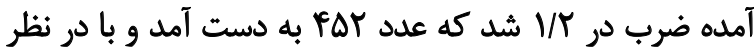

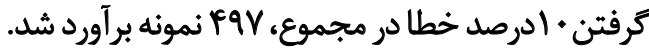

$Z=1 / 96(p 0 / 05)$

$d=0 / 05$ (power of the study \%95)

$P=\% 43$ (valid study)

$n=377$

$n \times 1 / 2=377 \times 1 / 2 \sim 452$

Final sample size $=452+(452 \times \% 10)=497$

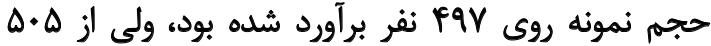

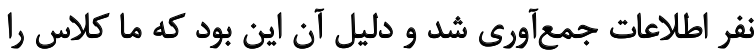
اندازهيرى كرده بوديم و تعداد دانش آموزان در كلاس آنها يكسان ئلان

\section{أزمودنى (أمنا}

مطالعه حاضر به روش مقطعى و روى ه.ه نفر از دانش آموزان

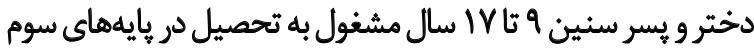

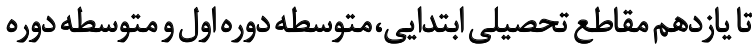

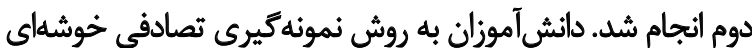

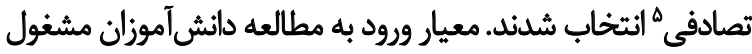

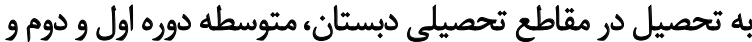

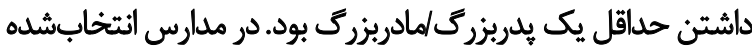

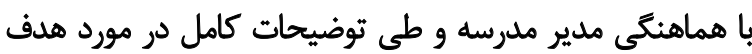

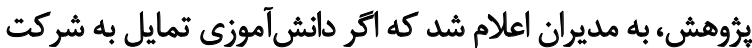

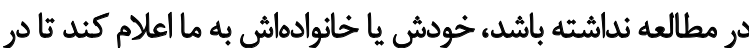

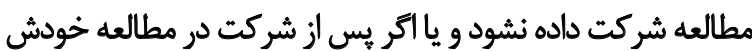

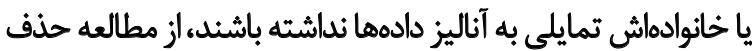

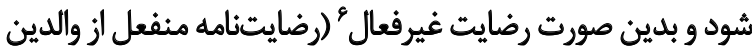

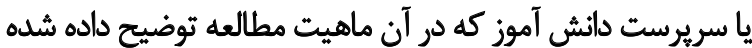
باشد) از دانش آموزان كرفته شد.

5. Cluster random sampling

6. Passive consent
زندكى سالمندان بر روند تكاملى نوههانيز تأثير داردو باعث سلامت إمت إمبت

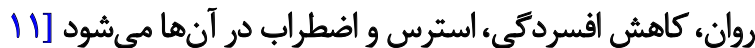

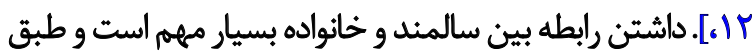

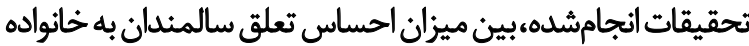

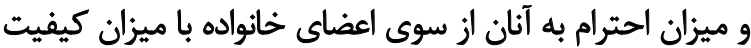
زندكى آنان ارتباط وجود دارد [ [II]].

خانواده بهعنوان يك منبع حمايتى حياتى به افراد سالمند

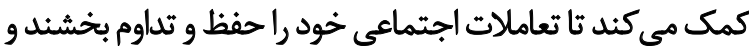

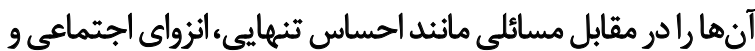

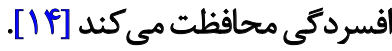

رابطه به هر نوع وابستكى و ارتباط ميان دو يا جند جيز و و تأثير

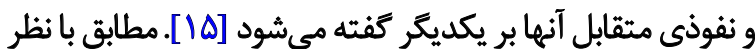

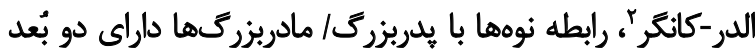

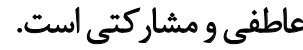

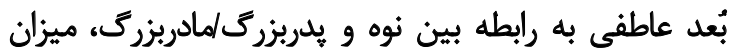

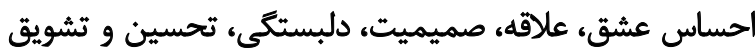

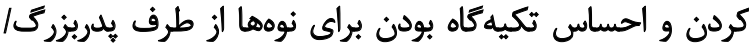

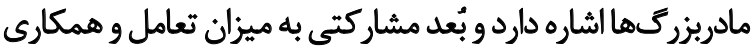

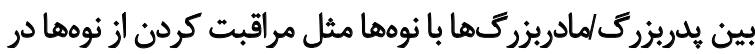

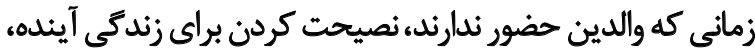

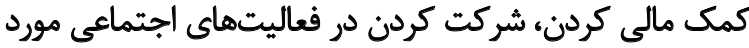

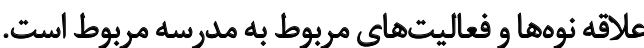

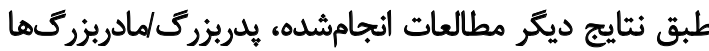

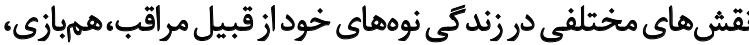

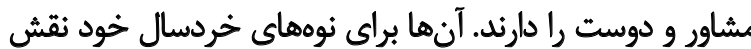

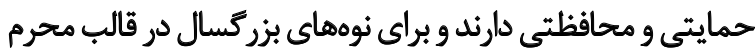

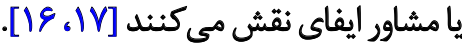

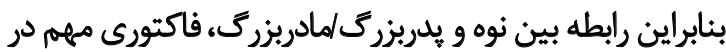

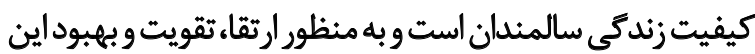

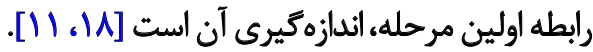

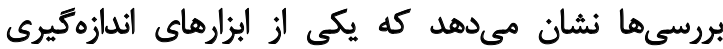

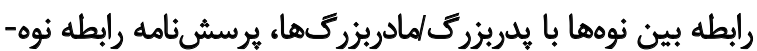

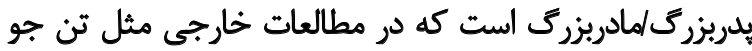

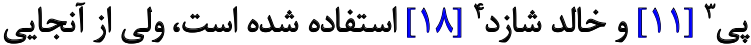

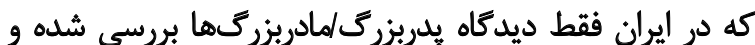

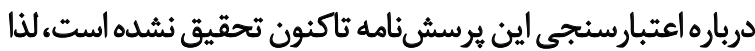

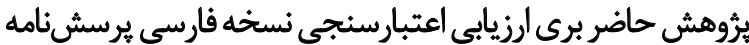

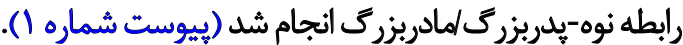

4. Khalid Shazia 
مسئله اصلى در روايى صورى، درك آزمودنى از مفهوم آزمون

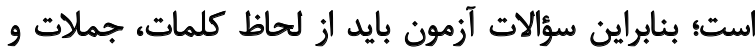

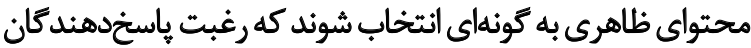

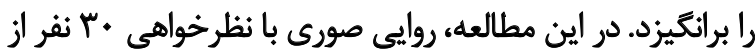

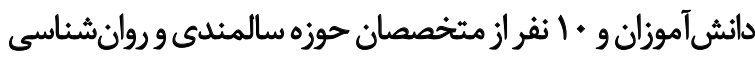

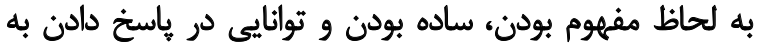

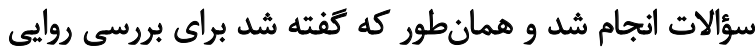

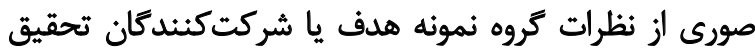
استفاده مي شُود ازئ نظرات

روايي سازه"

در اين يُوهش براي تعيين روايى سازه از روش تحليل عاملى

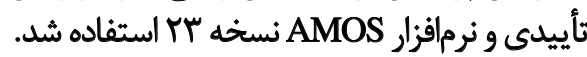

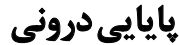

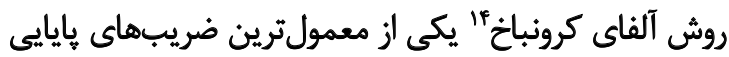

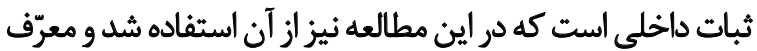

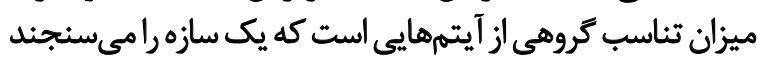

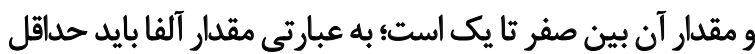

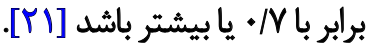

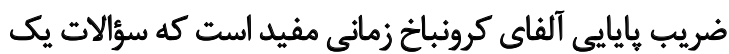

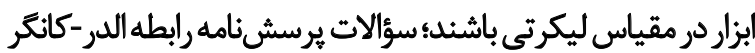
در طيف ليكرتى بودند.

بإيلوتمطالعه

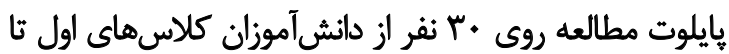

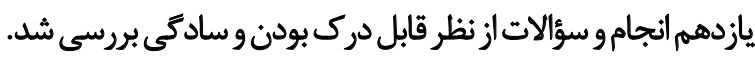

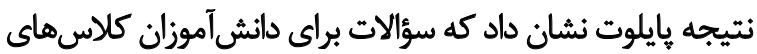

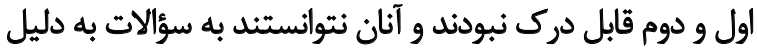

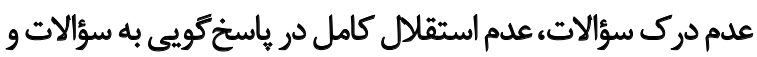

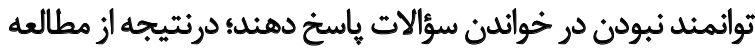

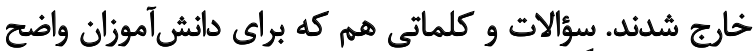
نبودند، مجدداً بررسى و واصلات و كلمانى هند.

يافتهنها

نمونها

ميانكين سنى دانشآموزان شركتكنئد در يروهش I IMA

12. Face Validity

13. Construct Validity

14. Cronbach's alpha

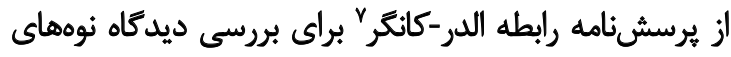

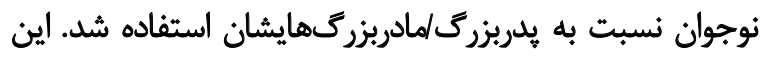

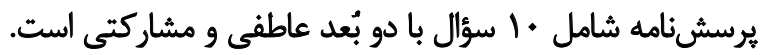

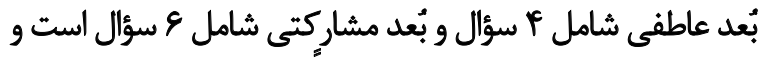

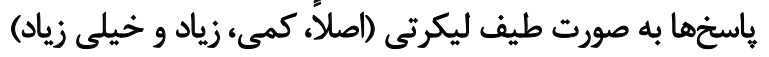

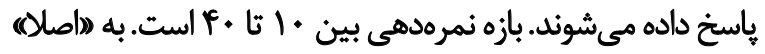

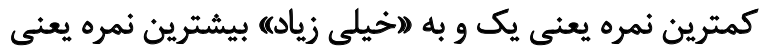
جهار داده مي نمود. فرآيندثرجمه

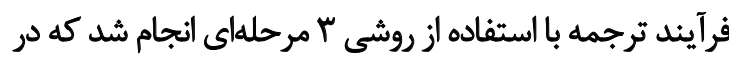

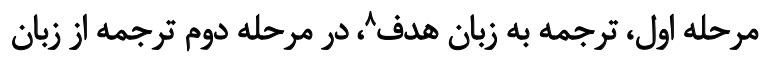

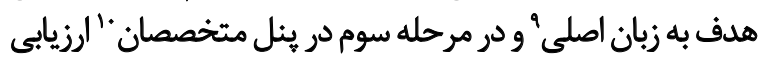

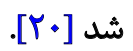

در مرحله اول دو نفر كه به زبان فارسى و انكليسى مسلط بودند

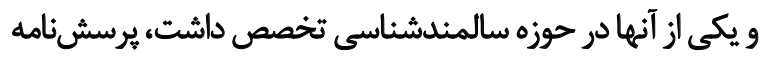

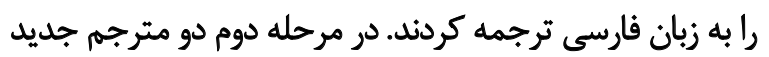

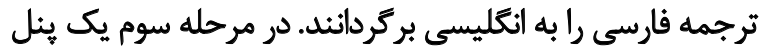

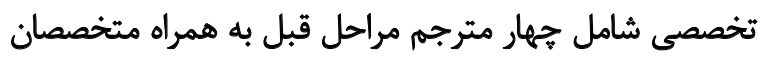

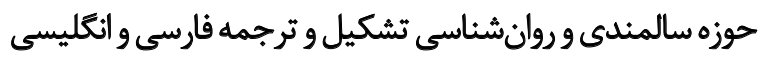

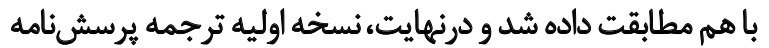
براى روايى صورى بررسى شد. روايي و ياياييى ثبرسشنامه

\section{روايي محتوا"}

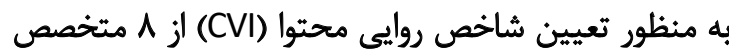

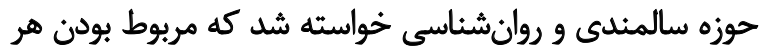

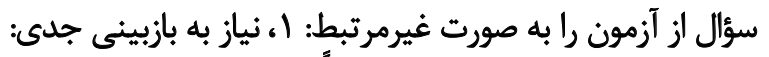

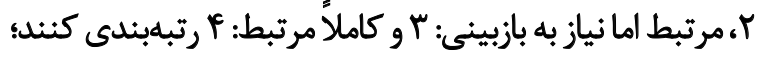

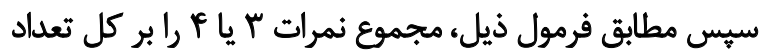
افراد متخصص تقسيم كرديم.

$$
\text { CV1 }
$$

7. Grandparent-Grandchild Relationship Scale (Elder \& conger, 2000)

8. Forward translation

9. Backward translation

10. Expert committee

11. Content Validity 


$$
\text { روايى سازه }
$$

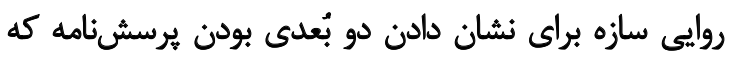

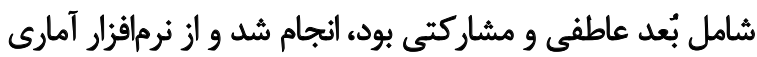

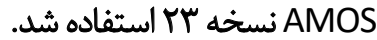

10GFI براى بررسى مناسب بودن برازش مدل از شاخصهاي AGFI، ' 'RMSEA

$G F I=0 / 964 ، C M I N=2 / 504 ، A G F I=0 / 938 ،, R M S E A=$ $0 / 059$

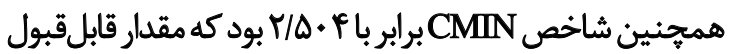

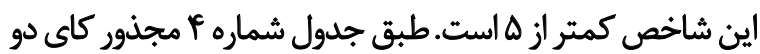

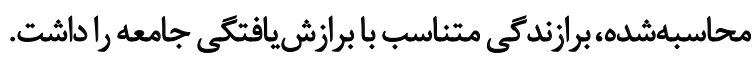
مطابق جدول شماره ه همه آيتمها بافاكتور مربوطهشان به طور

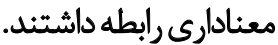

بإيايي

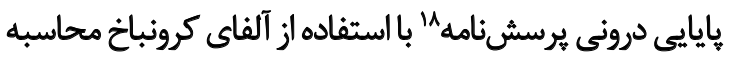

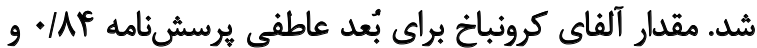

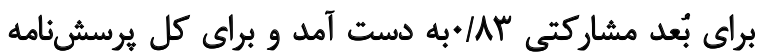

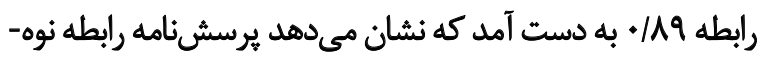

15. Goodness-of-fit index

16. Adjusted goodness-of-fit index

17. Root-mean-square error of approximation

18. Internal consistency

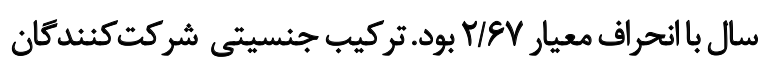

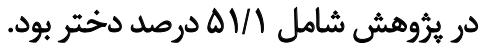

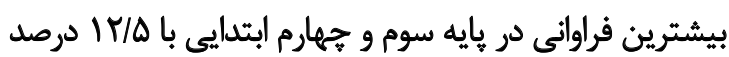

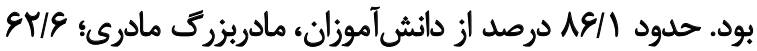

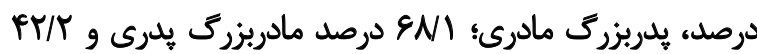

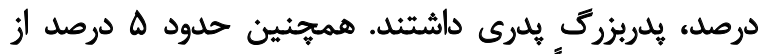

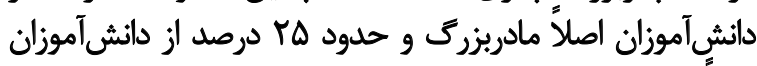
اصلاً يلربزرئ نداشيتيند.

$$
\text { رواليى مهتوا }
$$

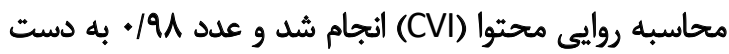

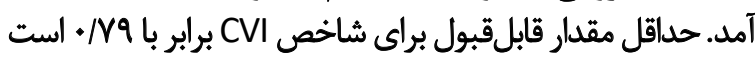

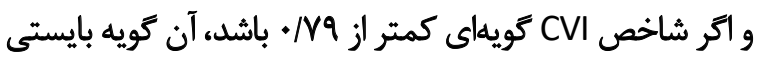

حذف شود [Tr]

مقادير بهدستآمده براي شاخص CVI نشان داد كه سؤالات

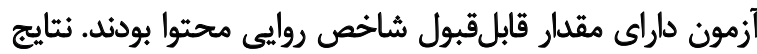
CVI

$$
\text { روايي صورى }
$$

دانشآموزان همه آيتمهاى يرسشنامه رابطه نوهإيدربزرى

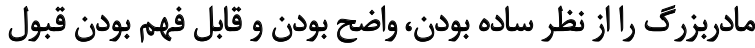

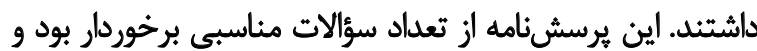

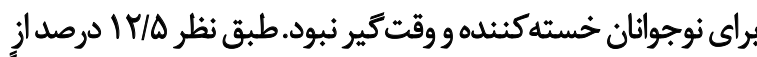

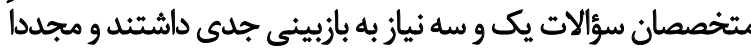

\begin{tabular}{|c|c|c|}
\hline $\mathrm{CVI}$ & تعداد متخصصانى كه به مربوط بودن آيتم نمره ب يا ع دادهاند & 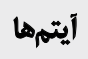 \\
\hline 1 & $\wedge$ & 1 \\
\hline 1 & 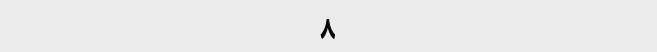 & $r$ \\
\hline +/AVa & $\checkmark$ & r \\
\hline 1 & $\wedge$ & $r$ \\
\hline 1 & $\wedge$ & $\Delta$ \\
\hline 1 & $\wedge$ & 8 \\
\hline 1 & $\wedge$ & $\checkmark$ \\
\hline 1 & $\wedge$ & $\wedge$ \\
\hline 1 & $\wedge$ & $q$ \\
\hline -/AVA & $\checkmark$ & 1. \\
\hline
\end{tabular}

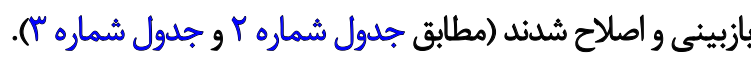

جدول ا. مقادير CVI بهدست آمده بر اساس نظرات متخصصان نمرهكذار 
جدول r. مربوط به ساده بودن روايى صورى

\begin{tabular}{|c|c|c|c|c|}
\hline \multicolumn{5}{|c|}{ ساده بودن } \\
\hline كاملأ ساده است & ساده است، اما نياز به بازيينى دارد & نياز به بازيينى جدى دارد & ساده نيست & سؤالات \\
\hline r & $r$ & 1 & • & 1 \\
\hline$\Delta$ & $r$ & - & • & r \\
\hline$\Delta$ & $r$ & 1 & • & $r$ \\
\hline $\mathrm{V}$ & 1 & $\cdot$ & • & r \\
\hline$\Delta$ & $r$ & $\cdot$ & $\cdot$ & $\Delta$ \\
\hline$\varepsilon$ & $r$ & $\cdot$ & • & 8 \\
\hline$r$ & $\Delta$ & . & . & $r$ \\
\hline$F$ & if & . & . & $\Lambda$ \\
\hline$r$ & 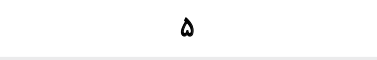 & $\cdot$ & - & 9 \\
\hline$\Delta$ & $r$ & . & . & 1. \\
\hline
\end{tabular}

جدول ب. مربوط به واضح بودن روايى صورى

\begin{tabular}{|c|c|c|c|c|}
\hline \multicolumn{5}{|c|}{ واضح بودن } \\
\hline كامالً واضع است & واضح استم اما ئياز به بازيينى دارد & نياز به بازيينى جلى دارد & واضح نيست & سؤالات \\
\hline$\Delta$ & $r$ & 1 & . & 1 \\
\hline$\Delta$ & $r$ & . & • & $r$ \\
\hline$r$ & $r$ & 1 & . & r \\
\hline$\Delta$ & $r$ & . & . & $f$ \\
\hline$r$ & $f$ & . & . & $\Delta$ \\
\hline 8 & $r$ & . & . & 8 \\
\hline$\Delta$ & $r$ & . & • & $\checkmark$ \\
\hline$f$ & f & . & . & $\wedge$ \\
\hline$f$ & $f$ & . & . & 9 \\
\hline$\Delta$ & $r$ & . & . & 1. \\
\hline
\end{tabular}

مادبزرك از شاخصهاي روايى محتواء روايى صورى، رواييى سازه

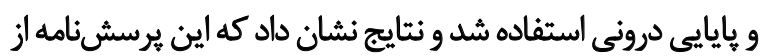
روايى و هايايى مناسبى برخوردار است.

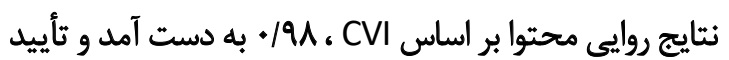

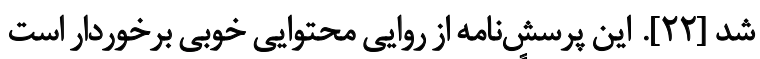

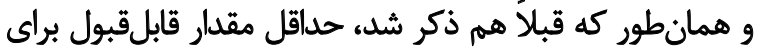

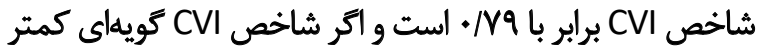

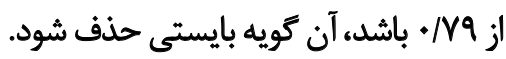

نتايج روايي صورى نشان داد كه سؤالات براى دانش آموزان از نظر سادگى، واضح بودن و قابل فهمه بودن مناسب است و وآنان
يدربزرى /مادربزرى از يايايى درونى مناسبى برخودار است.

ب

مطالعه حاضر با هدف ارزيابي اعتبارسنجى نسخه فارئي

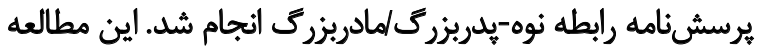

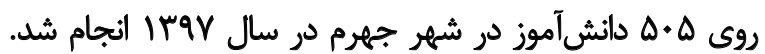

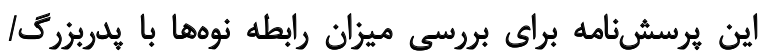

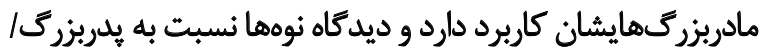
مادربزركهايشان راندازمكيرى مى كنداند

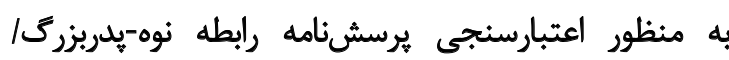




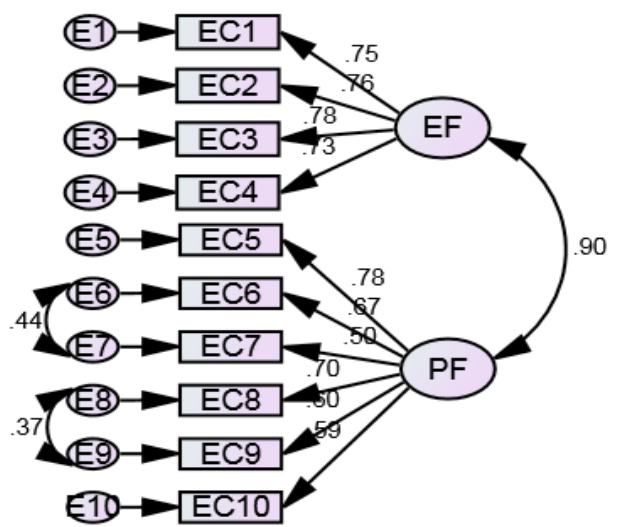

Elder Conger Scale:

EF: Emotion Factor

PF: Participation Factor

CMIN $(<5)=2.504 ; \operatorname{GFI}(>.9)=.964$

$\mathrm{AGFI}(>.9)=.938 ; \operatorname{RMSEA}(<.08)=.059$

شكل ا. ساختار تك عاملى برسشئامه رابطه نوه-بدربزرى /مادربزرى ( الدر-كانتر)

جدول f. شاخصهاى برازش تحليل عامل ثأييدى رابطه نوه-بدريزرى لمادربزرى ( الدر-كانكر)

\begin{tabular}{|c|c|c|}
\hline ارزش هاي محاسبهشيده مدل & ارزش هاي مورد انتظار & شاخصص مناسبت مدل \\
\hline$r / \Delta+F$ & $\Delta \leq$ & CMIN \\
\hline ./वसA & $5 . / q$ & AGFI \\
\hline .1984 & $\leq \cdot / 9$ & GFI \\
\hline .1 .09 & $.1 \cdot 1 \leq$ & RMSEA \\
\hline
\end{tabular}

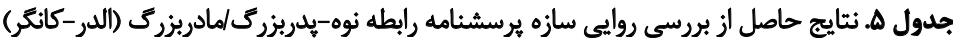

\begin{tabular}{|c|c|c|c|c|c|}
\hline \multirow{2}{*}{$\begin{array}{c}\text { GM } \\
\text { GM_M4 }\end{array}$} & \multicolumn{2}{|c|}{$\mathrm{EF}$} & \multirow{2}{*}{$\begin{array}{c}\text { Estimate } \\
1 / \ldots\end{array}$} & \multirow[t]{2}{*}{ S.E. } & \multirow[t]{2}{*}{ C.R. } \\
\hline & $<-$ & EF & & & \\
\hline GM_M3 & $<--$ & $\mathrm{EF}$ & $1 / \cdot 18$ &.$/ * r$ & $\mid Q / / V T$ \\
\hline GM_M2 & $<--$ & $\mathrm{EF}$ & - /AAQ &.$/ . \Delta A$ & $\mid F / A \Delta \Delta$ \\
\hline GM_M1 & $<--$ & $\mathrm{EF}$ & $1 / \cdot w$ &.$/ n$ & $I F / v \cdot V$ \\
\hline GM_M10 & $<--$ & PF & $1 / \ldots$ & & \\
\hline GM_M9 & $<--$ & PF & $\cdot / A \cdot V$ &.$/ \cdot 11$ & १/Q४ \\
\hline GM_M8 & $<--$ & PF & $1 / . .8$ & .1 .9$. & W/IFV \\
\hline GM_M7 & $<-$ & PF & $\cdot / 11 \cdot 9$ & .1 .94 & NV.. \\
\hline GM_M6 & $<--$ & PF & IVET & .1 .97 & $1 . M r r$ \\
\hline GM_M5 & $<--$ & PF & $1 / \cdot r V$ &.$/ \cdot A Y$ & II/AY \\
\hline
\end{tabular}

㿠 


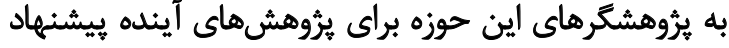

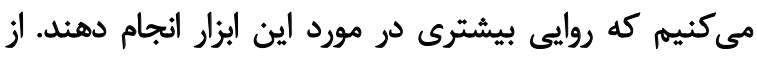

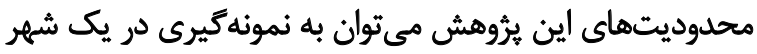

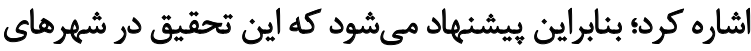

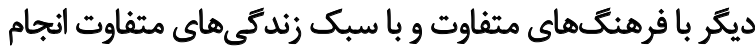

\section{تثيجهَيرى نمهايي}

نتايج حاصل از مطالعه حاضر نشان داد كه نسخه فارسى

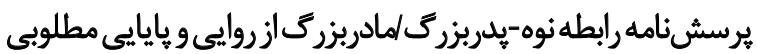

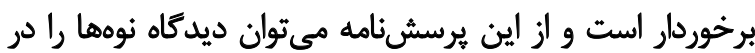

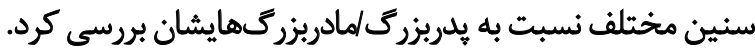

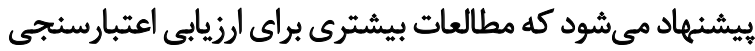

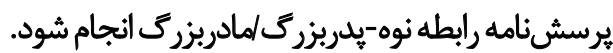

\section{ملاحظات اخلاقي \\ يبروى ازٔ اصول الخلاق يثوهش}

كميته اخلاق دانشكاه علوم بهزيستى و توانبخشى اين مقاله

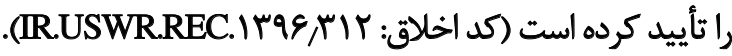

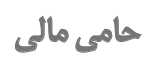

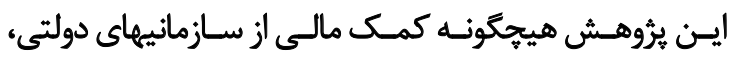

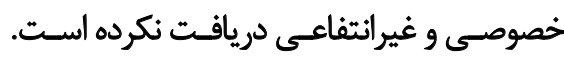

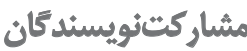

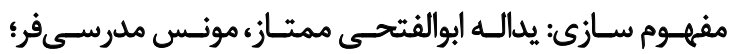

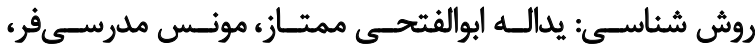

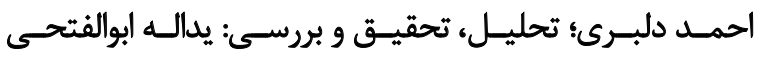

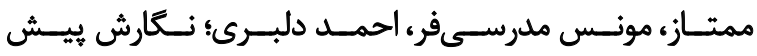

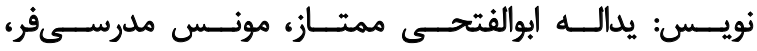

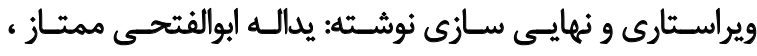

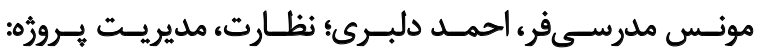

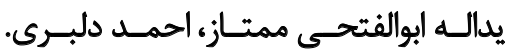

$$
\text { تعارض مثاقع }
$$

بنابر اظهار نويسندكان، اين مقاله تعارض منافع ندارد.
مىتوانند بهراحتى و بدون مشكل به آنها ياسخ دهند و اين سؤالات مناسب سنين 9 سال به بالاست.

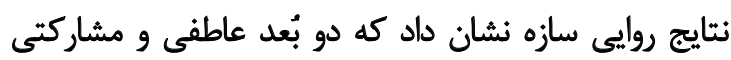

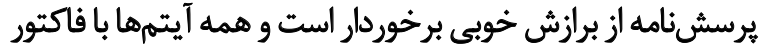
مربوطهان به طور معنادارى رابطه داشتند. برخد

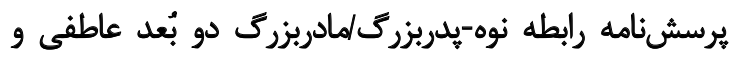

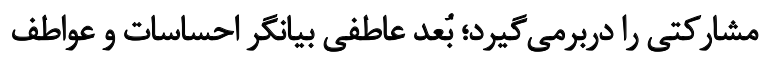

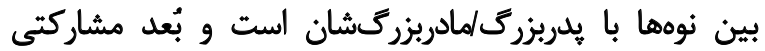

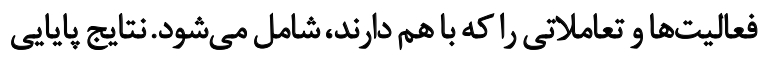

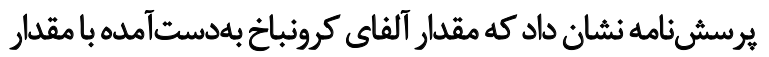

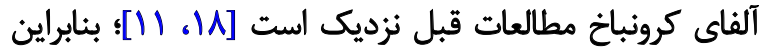
مي توان كفت كه اين يرسشنامه مياياست

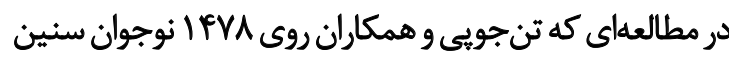

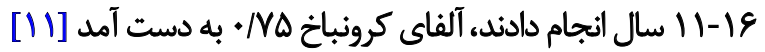

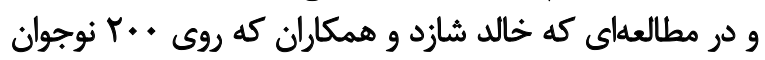

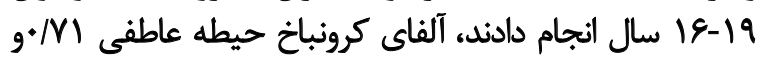

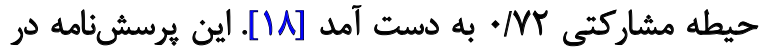

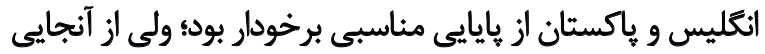

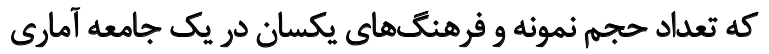

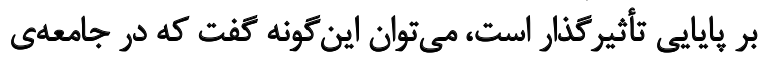

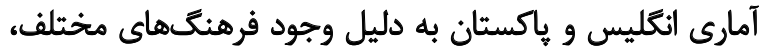

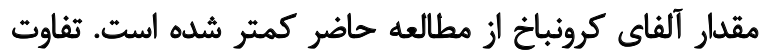

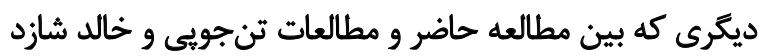

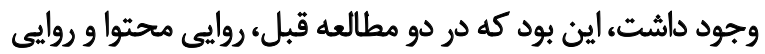

صورى محاسبه نشده بود.

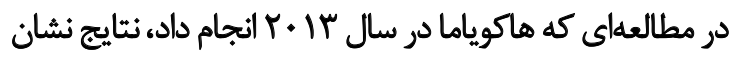

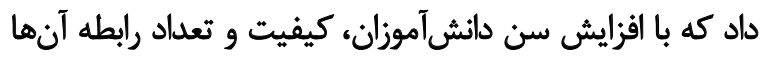

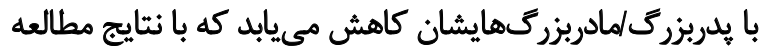

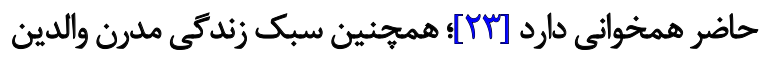

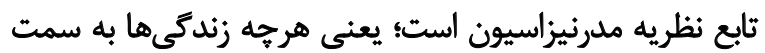

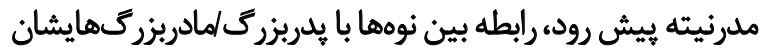

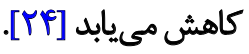

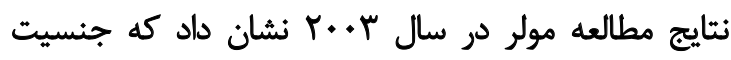

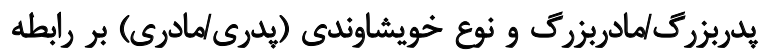

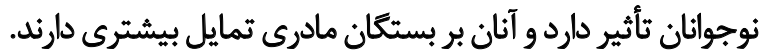

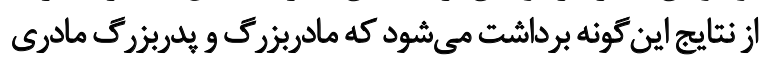

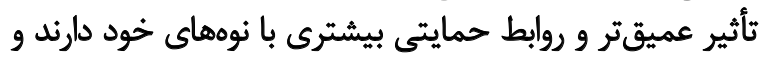

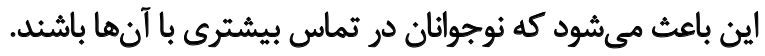

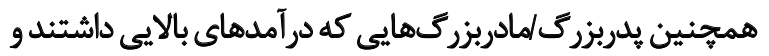

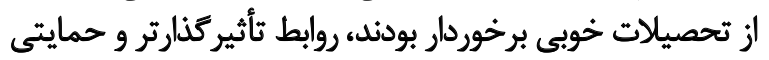

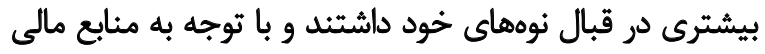

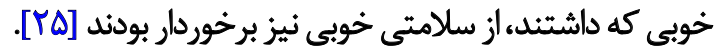




\section{References}

[1] Maghsoudnia S, Shojaei H. General elderly and senior medicine. Tehran: Veterans engineering and medical sciences research institute; 2005.

[2] Kosheshi M, Khosravi A, Alizadeh M, Torkashvand M, Aghaei N. Population aging in IR Iran, Socio-economic, Demographic and Health Characteristics of The Elderly: Issues and Challenges Tehran, Iran [Internet]. 2013 [Cited 2013 Jun 15]. Available from: https://iran.unfpa.org/en

[3] Statistics Center. General results of Population and Housing Census 2016-2017. [Internet]. 2017 [Cited 2017 Jun 15]. Available from: https:/www.amar.org.ir.

[4] Zarghami H. An review of ageing dynamics in IRAN. Tarbiat Modares University. 2006; 3(6):73-94

[5] Afsharkohan J, Koolivand S. Structured study of the quality of life in the elderly in Iran (2004-2013). Iranian Journal of Ageing. 2015; 10(3):192-201

[6] Pashos A. The evolutionary versus socio-economic view on grandparenthood: What are the grandparents' underlying motivations? Behavioral and Brain Sciences. 2010; 33(1):33-4 [DOI:10.1017/S0140525X09991713]

[7] Rowe JW, Kahn RL. Successful aging. The Gerontologist. 1997; 37(4):433-40. [DOI:10.1093/geront/37.4.433] [PMID]

[8] Johnson RA. Traditions in a new time: Stories of kinship foster grandmothers. New York: Columbia University; 1995.

[9] Habibi Sula A, Nikpour S, Sayyid al-shahadaee M, Haqqani H. Health promoting behaviors and related factors in the elderly. Iranian Journal of Nursing. 2006; 19(47):35-48.

[10] Monserud MA. Intergenerational relationships and affectual solidarity between grandparents and young adults. Journal of Marriage and Family. 2008; 70(1):182-95. [DOI:10.1111/j.17413737.2007.00470.x]

[11] Tan JP, Buchanan A, Flouri E, Attar-Schwartz S, Griggs J. Filling the parenting gap? Grandparent involvement with UK adolescents. Journal of Family Issues. 2010; 31(7):992-1015. [DOI:10.1177/0192513X09360499]

[12] Ruiz SA, Silverstein M. Relationships with grandparents and the emotional wellbeing of late adolescent and young adult grandchildren. Journal of Social Issues. 2007; 63(4):793-808. [DOI:10.1111/j.1540-4560.2007.00537.x]

[13] Yazdanpour F, Samaram A. Effective factors on life quality of elderly in Khomein. Social Development and Welfare Planning. 2011;3(6):45-63.

[14] Murphy E. Social origins of depression in old age. The British Journal of Psychiatry. 1982; 141(2):135-42. [DOI:10.1192/ bjp.141.2.135] [PMID]

[15] Shoaari Nejad AA. Farhang Olom Raftari. Tehran: Amirkabir; 1995.

[16] Attar-Schwartz S, Tan JP, Buchanan A, Flouri E, Griggs J. Grandparenting and adolescent adjustment in two-parent biological, lone-parent, and step-families. Journal of family psychology. 2009; 23(1):67. [DOI:10.1037/a0014383] [PMID]
[17] Silverstein M, Marenco A. How Americans enact the grandparent role across the family life course. Journal of Family Issues. 2001; 22(4):493-522. [DOI:10.1177/019251301022004006]

[18] Khalid S, Ahmed F, Imdad M. Grandparenting and adolescents' personality development. International Proceedings of Economics Development and Research. 2012; 48:143-7.

[19] Esmaeili M, Etemadar N, Negahdari M, editors. The prevalence of behavioral disorders among primary school students and their relationship with parents' reports (Case Study of Estahban). 6th International Congress on Child and Adolescent Psychiatry. Tabtiz: Tabtiz university of medical sciences; 2013 Sep 16-18; Tabtiz, Iran.

[20] Tsang S, Royse CF, Terkawi AS. Guidelines for developing translating, and validating a questionnaire in perioperative and pain medicine. Saudi Journal of Anaesthesia. 2017; 11(Suppl 1):S80. [DOI:10.4103/sja.SJA_203_17] [PMID] [PMCID]

[21] Mohammadbeigi A, Mohammadsalehi N, Aligol M. Validity and reliability of the instruments and types of measurments in health applied researches. Journal of Rafsanjan University of Medical Sciences. 2015; 13(12):1153-70.

[22] Hosseini Z, Ghorbani Z, Ebn Ahmady A. Face and content validity and reliability assessment of change cycle questionnaire in smokers. Journal of Mashhad Dental School. 2015; 39(2):147-54

[23] Hakoyama M, MaloneBeach EE. Predictors of grandparentgrandchild closeness: An ecological perspective. Journal of Intergenerational Relationships. 2013; 11(1):32-49. [DOI:10.1080/15350 770.2013.753834]

[24] Durkheim E. The division of labor in society. Trans WD Halls New York, NY: Free Press. 1893.

[25] Mueller MM, Elder GH. Family contingencies across the generations: grandparentgrandchild relationships in holistic perspective. Journal of Marriage and Family. 2003; 65(2):404-17. [DOI:10.1111/j.1741-3737.2003.00404.x] 
يويوست ا. يرسش نامه رابطه نوه-بدربزرك/مادربزرى

\begin{tabular}{|c|c|c|c|c|c|}
\hline خيلى زياد & زياد & كمي & اصلأ & 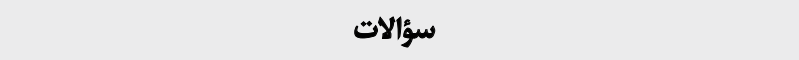 & رديف \\
\hline & & & & تا جه اندازه مى توانيد به يلريزرى /مادربزركتان تكيه كنيد؟ & 1 \\
\hline & & & & تا جه انلازه احساس مى كنيد يلربزرى /مادربزرى تان شما رادوست دارد؟ & r \\
\hline & & & & ثأ جه اندازه يلربزرى /مادربزرى تان شما را در انجام كارها تشويق مىكند؟ & r \\
\hline & & & & تا جها اندازه (در مقايسه با ديكر نومها) با بلربزرى /مادربزركتان صميمى هستيد؟ & f \\
\hline & & & & 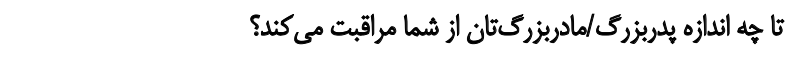 & $\Delta$ \\
\hline & & & & مي جاهي اندازه هدربزرى/مادربزركتان در فعاليتهاى اجتماعى مورد علاقه شما شركت & 8 \\
\hline & & & & 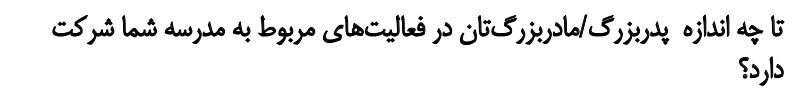 & $r$ \\
\hline & & & & 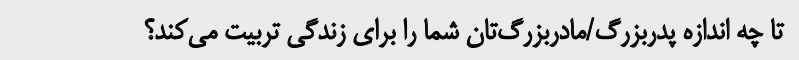 & $\wedge$ \\
\hline & & & & 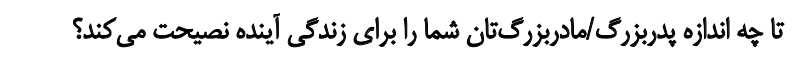 & $q$ \\
\hline & & & & 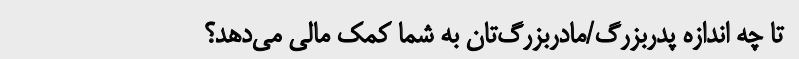 & 1. \\
\hline
\end{tabular}

